To appear in "Magnetic Coupling between the Interior and the Atmosphere of the Sun", eds. S. S. Hasan and R. J. Rutten, Astrophysics and Space Science Proceedings, Springer-Verlag, Heidelberg, Berlin, 2009.

\title{
The Evershed Effect with SOT/Hinode
}

\author{
K. Ichimoto ${ }^{1}$ and the SOT/Hinode Team \\ Kwasan and Hida Observatories, Kyoto University, Japan
}

\begin{abstract}
Summary. The Solar Optical Telescope onboard Hinode revealed the fine-scale structure of the Evershed flow and its relation to the filamentary structures of the sunspot penumbra. The Evershed flow is confined in narrow channels with nearly horizontal magnetic fields, embedded in a deep layer of the penumbral atmosphere. It is a dynamic phenomenon with flow velocity close to the photospheric sound speed. Individual flow channels are associated with tiny upflows of hot gas (sources) at the inner end and downflows (sinks) at the outer end. SOT/Hinode also discovered "twisting" motions of penumbral filaments, which may be attributed to the convective nature of the Evershed flow. The Evershed effect may be understood as a natural consequence of thermal convection under a strong, inclined magnetic field. Current penumbral models are discussed in the lights of these new Hinode observations.
\end{abstract}

\section{Introduction}

Since its discovery by Evershed (1909), the Evershed effect has been one of the longstanding mysteries in solar physics. It is evident from recent high-resolution observations that the flow is closely related to the smallscale filamentary structures in penumbrae (for reviews see Solanki 2003, Thomas et al. 2004, 2008, Bellot Rubio 2007, Thomas 2009). The inclination of the penumbral magnetic field fluctuates in the azimuthal direction with the spatial scale of the penumbral filaments (interlocking comb structure), whereas the flow takes place in radial filaments which have nearly horizontal magnetic field (Degenhardt \& Wiehr 1991, Schmidt et al. 1992, Title et al. 1993). The flow vector is parallel to the magnetic fields (Bellot Rubio et al. 2004, Borrero et al. 2005).

To account for the filamentary structure of penumbrae, the following models have been proposed, i.e., the embedded or rising flux tube model Solanki \& Mondavon 1993, Schlichenmaier et al. 1998) in which the Evershed flow channels are explained as rising flux tubes embedded in more 
vertical background magnetic fields in the penumbra, the gappy penumbral model (Spruit \& Scharmer 2006, Scharmer \& Spruit 2006) in which the bright penumbral filaments are regarded as manifestations of the protrusion of non-magnetized, convecting hot gas into the oblique background fields of the penumbra, and the downward pumping model (Thomas et al. 2002, Weiss et al. 2004, Brummell et al. 2008) in which the penumbral fine structure is created by localized submergence of penumbral fields that are forced by the photospheric convection around the outer edge of penumbrae. There is still no consensus on the physical nature and origin of the penumbral fine structures.

Using highly stable time sequences of sunspot images and high precision spectropolarimetric data provided by the Solar Optical Telescope (SOT, Tsuneta et al. 2007, Suematsu 2007) onboard Hinode (Kosugi et al. 2007), Ichimoto et al. (2007a) confirmed the interlocking-comb penumbral structure and found that the Evershed flow preferentially takes place in bright filaments in the inner penumbra and in dark filaments in the outer penumbra. They also found the presence of a number of small patches with a vertical velocity component, upward motions distributed over the penumbra, and strong downward motions associated with magnetic polarity opposite to that of the sunspot in the mid and outer penumbra. The vertical motions may be regarded as the sources and sinks of the Evershed flow channels, though unequivocal identification of individual pairs was not reached.

Here we report results on the spatial distribution of vertical motions in penumbrae obtained from further analyses of the sunspot data taken by SOT. The characteristics of the Evershed flow are summarized, and the origin of the flow is discussed within the context of the present penumbral models.

\section{Elementary structure of the Evershed flow}

The spatial distribution of the vertical motions in sunspots were studied using SOT/SP data taken on May 1, 2007. The sunspot was located near disk center, with the viewing angle between the line of sight and the normal to the solar surface $\mu=5.8^{\circ}$. Stokes profiles of the pair of Fe I lines at $6302 \AA$ were fitted using the Milne-Eddington model to obtain vector magnetic fields.

Figure 1 shows the magnetic field inclination overlayed with contours showing the vertical motions. The blue contours show blueshift at $-0.8 \mathrm{~km} \mathrm{~s}^{-1}$ in the wing of Stokes- $I$ of Fe I $6301.5 \AA$ and the red countours show $V / I_{c}=0.01$ at Fe I $6302.5 \AA+0.365 \AA$, representing strong downflow regions with opposite magnetic polarity to the spot. $V$ and $I_{c}$ are Stokes- $V$ and the continuum intensity, respectively. Radial penumbral filaments with dark appearance in the inclination map are the channels with nearly horizontal magnetic field. It is remarkable that the upflow and downflow patches are aligned with the horizontal field channels that carry the Evershed flow, and that small-scale upflows are preferentially located near the inner ends, downflows at the outer 


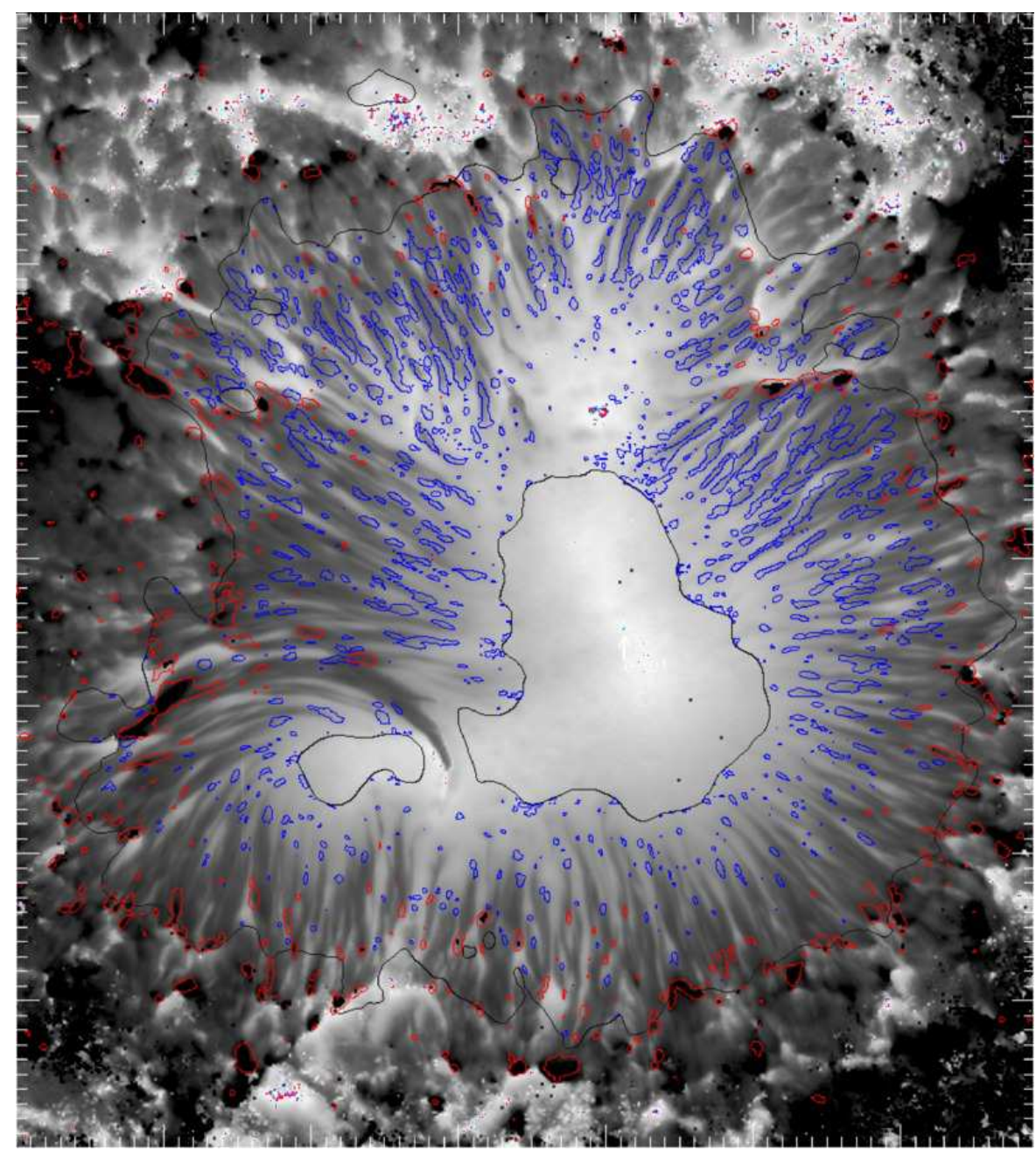

Fig. 1. Inclination of the magnetic field. This sunspot was observed by SP/SOT on May 1, 2007. Top is toward the disk center; the heliocentric distance is $5.8^{\circ}$. The black contours show the inner and outer boundaries of the penumbra. Dark channels in the penumbra represent nearly horizontal fields. Overlaid are contours showing the vertical motions, i.e., blue for upflow and red for downflow. 


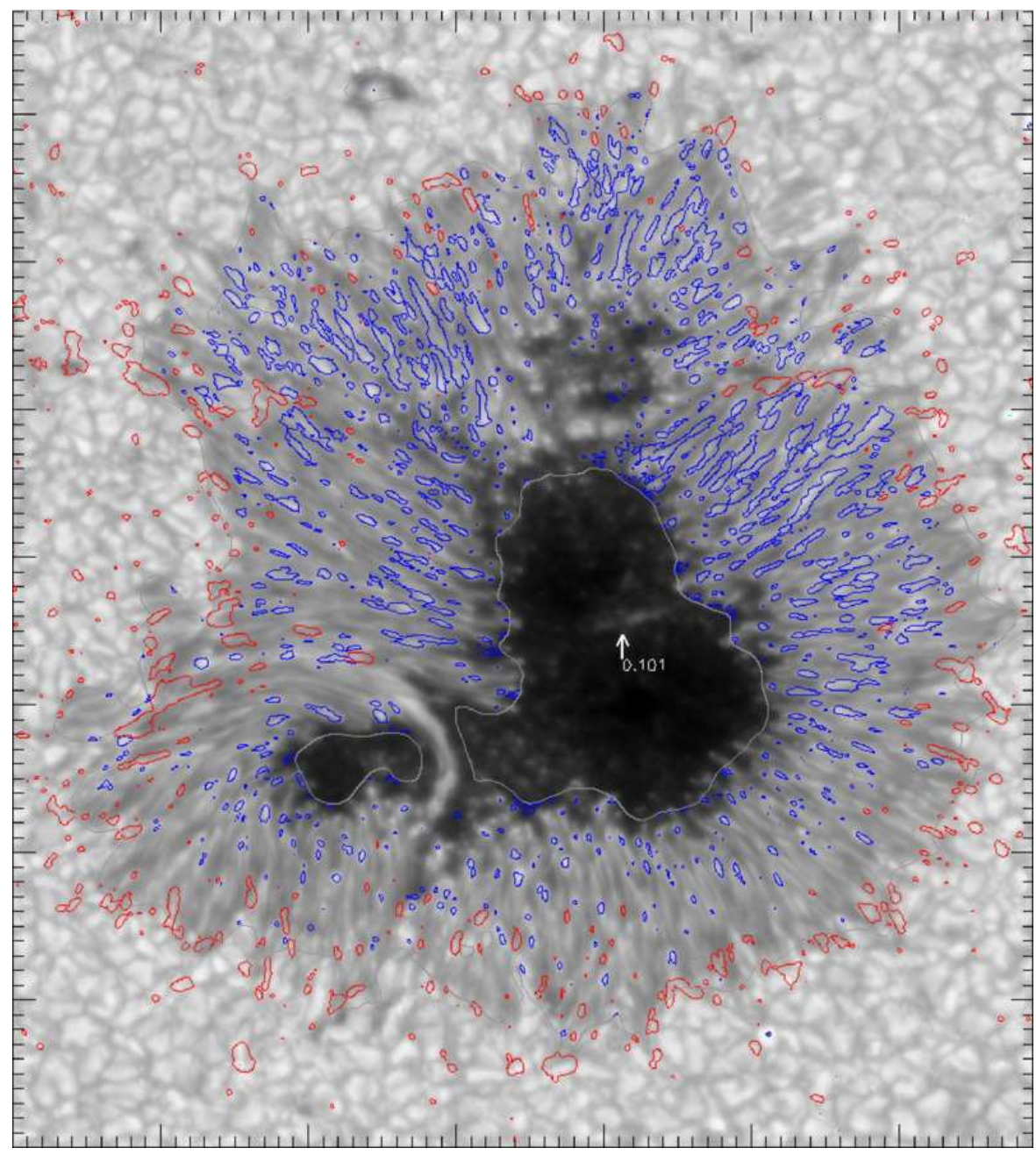

Fig. 2. The same sunspot in continuum intensity near $\lambda=630 \mathrm{~nm}$, with the same contours as in Fig. 1 .

ends of the horizontal field channels. Thus, the upflow and downflow patches may be regarded as the sources and sinks of the Evershed flow elements, embedded in the deep penumbral photosphere. It is noted that the Stokes-V signal is well visible even at FeI $6302.5 \AA+0.454 \AA\left(22 \mathrm{~km} \mathrm{~s}^{-1}\right)$. This suggests the presence of supersonic flows near the outer end of the flow channels (see Ichimoto et al. 2007a).

Figure 2 shows the continuum intensity of the sunspot overlayed with the same contours as in Fig. 1. The spatial correlation between bright grains and upflows is remarkable. It is obvious that the Evershed (up-)flow carries the 
energy to maintain the penumbral brightness, although quantitative evaluation of the energy flux carried by the upflow is not straightforward because the line forming layer is likely well above the vigorously convectiving layer.

\section{Discussion}

Ichimoto et al. (2007b) found a "twisting" motion in the leading part of the bright filaments in penumbrae. Since the visibility of this twisting motion depends on the viewing angle, i.e., it is seen only when the penumbral filaments are observed from their side, Ichimoto et al. (2007b) interpreted the apparent twisting feature as a manifestation of overturning convection in the source region of the Evershed flow. Zakharov et al. (2008) confirmed the twisting motions of penumbral filaments with ground-based observations and concluded that the twisting features indicate the presence of convective rolls in filaments with a nearly horizontal magnetic field.

From the analysis of the net circular polarization in penumbrae, Ichimoto et al. (2008) found evidence of positive correlation between the Evershed flow velocity and the magnetic field strength, spatially along the line of sight, in the sense that the flow velocity and the magnetic field strength both increase with depth in the flow channels. This is supports the conclusion that the flowing gas is strongly magnetized. The Stokes- $V$ Dopplershift or the Dopplershift of polarized spectral-line components in penumbrae serves as more direct evidence that the flowing gas is not field-free.

The properties of the Evershed effect so far obtained are summarized as follows:

- Each Evershed flow channel is associated with upflow (source) and downflow (sink) in its inner and outer ends;

- The geometry of the magnetic field is consistent with low-lying arched tubes embedded in more inclined magnetic fields, i.e., so-called interlockingcomb penumbral structure;

- There is a very good spatial correlation between upflows and bright penumbral grains, which strongly suggests that the Evershed (up-)flow carries the energy for maintaining the penumbral brightness;

- The source region of the Evershed flow channels shows hints of overturning convection;

- The flow velocity (and the magnetic field strength) increase with depth in the visible layers of the flow channel;

- The flow plasma is not field-free but magnetized.

These characteristics obviously suggest a convective origin for the Evershed effect. Let us consider the currently representative penumbral models, i.e., the embedded-fluxtube model and the gappy model, in the light of these 
observational results. There is no observational evidence of the presence of a lower boundary of fluxtubes at least in the inner and middle penumbra. Thus, the picture of narrow fluxtubes in the embedded-fluxtube model is not supported by observation. On the other hand, the flowing gas is highly magnetized, which is an obvious contradiction between the field-free gap model and the observations. If the fluxtube model allows vertically elongated "flux tubes" (or slabs), and if the gap model discards the term "field free", then there is no fundamental difference between the two models. In both models, rising motions of hot gas are driven by buoyancy forces. These new observational results suggest that the Evershed effect can be understood as a natural consequence of thermal convection under strong, inclined magnetic fields.

Recent 3D numerical MHD simulations (Heinemann et al. 2007, Rempel et al. 2008) of sunspots have successfully reproduced the interlocking-comb structure of the magnetic fields in penumbrae with Evershed outflows along filaments with nearly horizontal magnetic field and overturning motions. Scharmer et al. (2008) argued that the Evershed flow represents the horizontal flow component of overturning convection in penumbrae, based on numerical simulations. The velocity of the Evershed outflow in current numerical simulations is, however, only a few $\mathrm{km} \mathrm{s}^{-1}$, which is much smaller than the observed one. The mechanism that drives the vigorous observed flow, especially at the outer end of the flow channel, is still to be identified. Siphon flow models (e.g., Thomas \& Weiss 1992) predict supersonic flows near the outer footpoint of the flow channel (Montesinos \& Thomas 1997), although the theory is based on a stationary model. A more dynamic picture is given by the downward pumping model (Weiss et al. 2004), in which stochastic flows may be driven by the convective collapse caused by the localized submergence of penumbral magnetic fields forced by the photospheric convection around the outer edge of penumbrae. The validity of these various models should be examined by investigating the temporal evolutions of the individual Evershed flow elements in further observations.

Acknowledgement. Hinode is a Japanese mission developed and launched by ISAS/JAXA, with NAOJ as domestic partner and NASA (USA) and STFC (UK) as international partners. It is operated by these agencies in co-operation with ESA and NSC (Norway). This work was carried out at the NAOJ Hinode Science Center, which is supported by the Grant-in-Aid for Creative Scientic Research, The Basic Study of SpaceWeather Prediction from MEXT, Japan (Head Investigator: K. Shibata), generous donations from Sun Microsystems, and NAOJ internal funding.

\section{References}

Bellot Rubio, L. R., Balthasar, H., Collados, M. 2004, A\&A, 427, 319

Bellot Rubio, L. R. 2007, Highlights Spanish Astrophys. IV, 271

Borrero, J. M., Lagg, A., Solanki, S. K., et al. 2005, A\&A, 436, 333 
Brummell, N. H., Tobias, S. M., Thomas, J. H., et al. 2008, ApJ, 686, 1454

Degenhardt, D., Wiehr, E. 1991, A\&A, 252, 821

Evershed, J. 1909, MNRAS, 69, 454

Heinemann, T., Nordlund, A., Scharmer, G. B., et al. 2007, ApJ, 669, 1390

Ichimoto, K., Shine, R. A., Lites, B., et al. 2007a, PASJ, 59, 593

Ichimoto, K., Suematsu, Y., Tsuneta, S., et al. 2007b, Science, 318, 1597

Ichimoto, K., Tsuneta, S., Suematsu, Y., et al. 2008, A\&A, 481, L9

Kosugi, T., Matsuzaki, K., Sakao, T., et al. 2007, Sol. Phys., 243, 3

Montesinos, B., Thomas, J. H., 1997, Nat, 390, 485

Rempel, M., Schuessler, M., Knölker, M. 2008, arXiv:0808.3294v2

Scharmer, G. B., Spruit, H. C. 2006, A\&A, 460, 605

Scharmer, G. B., Nordlund, A., Heinemann, T., 2008, ApJ, 677, L149

Schmidt, W., Hofmann, A., Balthasar, H., et al. 1992, A\&A, 264, L27

Schlichenmaier, R., Jahn, K., Schmidt, H. U. 1998, A\&A, 337, 897

Solanki, S. K. 2003, Astron. Astrophys. Rev. 11, 153

Solanki, S. K., Montavon, C. A. P. 1993, A\&A, 275, 283

Spruit, H. C., Scharmer, G. B. 2006, A\&A, 447, 343

Suematsu, Y., Tsuneta, S., Ichimoto, K., et al. 2008, Sol. Phys., 249, 197

Thomas, J. H., Weiss, N. O., 1992, in Sunspots; Theory and Observations, eds. J.

H. Thomas, N.O.Weiss, Kluwer, Dordrecht, 3

Thomas, J. H., Weiss, N. O., Tobias, S. M. et al. 2002, Nat, 420, 390

Thomas, J. H., Weiss, N. O. 2004, ARAA, 42, 517

Thomas, J. H., Weiss, N. O. 2008, Sunspots and Starspots, Cambridge University Press

Thomas, J. H. 2009, in Magnetic Coupling between the Interior and the Atmosphere of the Sun, eds. S. S. Hasan, R. J. Rutten, Astrophys. Space Sci. Procs., Springer, Heidelberg, these proceedings

Title, A., Frank, Z., Shine, R., et al. 1993, ApJ, 403, 780

Tsuneta, S., Ichimoto, K., Katsukawa, Y., et al. 2008, Sol. Phys., 249, 167

Weiss, N. O., Thomas, J. H., Brummell, N. H. et al. 2004, ApJ, 600, 1073

Zakharov, V., Hirzberger, J., Riethmuller, T., et al. 2008, A\&A, 488, L17 